\title{
Presencia de Serapias vomeracea Briq. (Orchidaceae) en Córdoba (Andalucía, España)
}

Soledad Carpintero Ortega, Francisco Jesús Cano Villegas

Área de Ecología, Departamento de Botánica, Ecología y Fisiología Vegetal, Universidad de Córdoba, España

\section{Correspondencia}

S. Carpintero

e-mail: solecarpintero@gmail.com

Recibido: 14 junio 2019

Aceptado:1 abril 2020

Publicado on-line: 24 abril 2020

Editado por: Marta Recio Criado

\section{Resumen}

Se confirma la presencia de Serapias vomeracea en la provincia de Córdoba (España).

Palabras claves: Orchidaceae, Serapias, Córdoba, España.

\begin{abstract}
Presence of Serapias vomeracea Briq. (Orchidaceae) in Cordoba (Andalusia, Spain)

The presence of Serapias vomeracea is confirmed in the province of Córdoba (Spain)
\end{abstract}

Key words: Orchidaceae, Serapias, Cordoba, Spain.
Flora Ibérica (Sáez et al., 2005) señala la presencia de Serapias vomeracea Briq. (Orchidaceae) en las provincias andaluzas de Málaga, Cádiz, Huelva y Córdoba y la Flora Vascular de Andalucía Occidental (Silvestre, 1987) la sitúa en Cádiz y Huelva. Sin embargo, otros autores (Benito \& Tabuenca 2001; Benito, 2014, 2017) dudan de su presencia en el sur peninsular y asimilan las citas de esta especie a Serapias strictiflora Welwitsch ex Veigaen.
En Andalucía, Becerra \& Robles (2009) y Benito (2017) reconocen 5 especies del género Serapias: Serapias parviflora Parl. y Serapias lingua L. ampliamente distribuidas; Serapias cordigera L. de distribución más limitada; $S$. strictiflora y $S$. vomeracea de distribución muy escasa. En la provincia de Córdoba, los últimos trabajos (Moreno et al., 1997; Becerra \& Robles 2009; López Tirado 2018) citan tres especies: S. lingua, S. parviflora y $S$. cordigera.

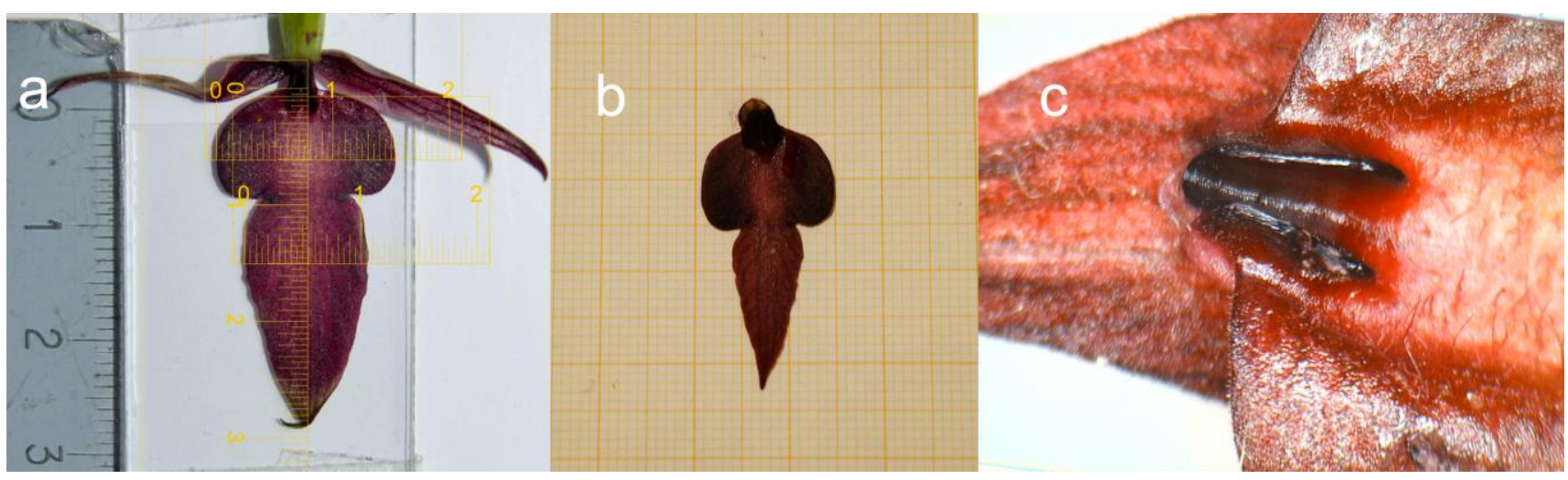

Figura 1. a) y b) Medidas de hipoquilo y epiquilo. c) Detalle de las callosidades.

Figure 1. a) y b) Hypochyle and epichile measures. c) Detail of callus. 
En el presente trabajo se ha localizado una población de $S$. vomeracea en la Sierra de Córdoba (Andalucía, España). Se recogió una muestra de flores y semillas que se encuentra depositada en el herbario de la Universidad de Córdoba (Pliego COFC-70741, recolección e identificación por Soledad Carpintero Ortega y Francisco J. Cano Villegas, 7 de mayo de 2019). Los caracteres que nos han permitido identificar el material recogido como S. vomeracea son los siguientes: base del labelo con dos callosidades, epiquilo con una longitud de 1,3 a 2,2 cm en las flores medidas $(n=4)$, la relación anchura/longitud del epiquilo está entre 0,38-0,45, coloración púrpura de las flores, tamaño que en algunos casos superaban los $40 \mathrm{~cm}$, y abundante y conspicua pilosidad cana del labelo (Benito, 2017; Sáez et al., 2005) (Figuras 1 y 2)

La población, con abundantes individuos, se localiza en una ladera próxima a un pastizal por el que discurre un arroyo temporal. No hemos encontrado ninguna otra orquídea en las inmediaciones de este

punto, aunque algo alejadas sí aparecen otras especies como Orchis langei K. Richt., Ophrys apifera Huds., Orchis italica Poir., Orchis champagneuxii Barnéoud, Ophrys ficalhoana J.A. Guim. y Ophrys tenthredinifera Willd.

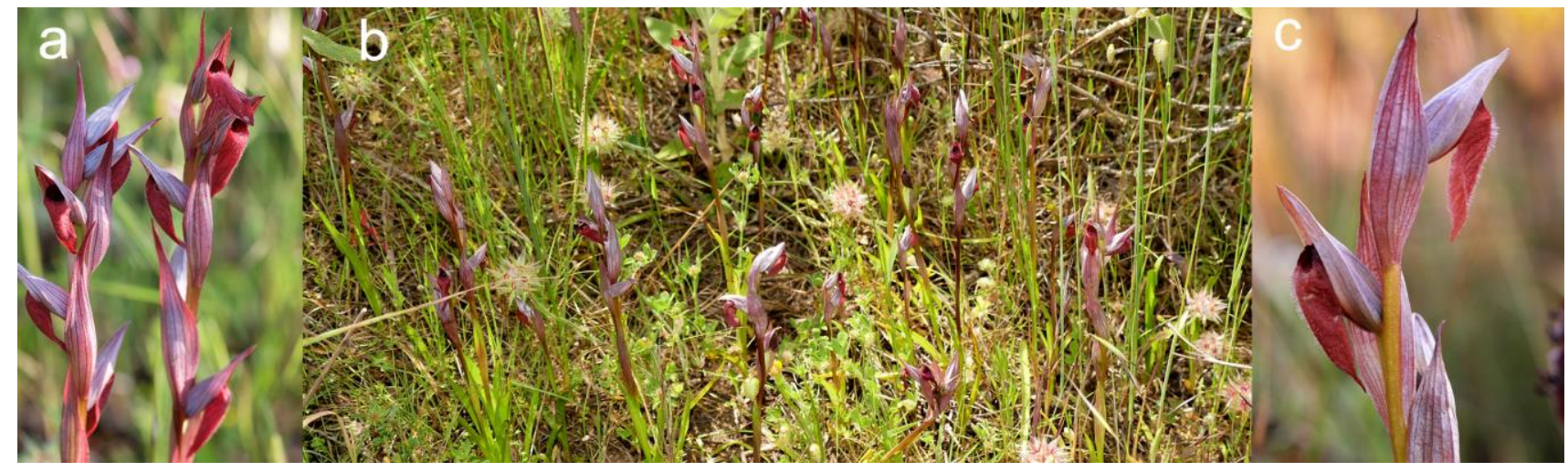

Figura 2. a) Pies de S. vomeracea. b) Vista general. c) Detalle de flores donde puede observarse la abundante pilosidad.

Figure 2. a) Specimens of S. vomeracea. b) General view. c) Detail of flowers and their abundant pilosity.

\section{Agradecimientos}

Queremos agradecer a Manuel Becerra y Javier Benito la ayuda prestada en la identificación de esta especie. Asimismo, agradecemos a los miembros del grupo facebook de Orquideas Silvestres de Andalucía sus muestras de interés y opiniones, especialmente a Javier Hernández, FerNan, Adolfo Ventas, AbouNabat, María José Kanito, Pablo Barrena, Mํㅡㄹ José Tarancón, Vicente Avola y Miguel Gómez. También agradecemos a Antonio Priego su ayuda en el campo, a Félix Infante y a un revisor anónimo su aporte a este trabajo.

\section{Bibliografía}

Becerra, M. \& Robles, E. (2009). Guía de campo de las orquídeas silvestres de Andalucía. Cádiz, Alcalá del Valle: La Serranía.

Benito Ayuso, J. (2014). Sobre la presencia de Serapias vomeracea (Burm. Fil.) Briq. en el Sistema Ibérico. Flora Montiberica, 57, 81-87.
Benito Ayuso, J. (2017). Estudio de las orquídeas silvestres del Sistema Ibérico (tesis doctoral), Departamento de Botánica y Geología, Universidad de Valencia, Valencia.

Benito Ayuso, J. \& Tabuenca, J.M. (2001). Apuntes sobre orquídeas ibéricas. Estudios del Museo de Ciencias Naturales de Álava, 16, 67-87.

López Tirado, J. (2018). Flora vascular del término municipal de Córdoba. Catálogo florístico y claves de identificación. Monografías de Botánica Ibérica, 22.

Moreno, B., Gómez, J. \& Infante, F. (1997). Recursos naturales de Córdoba. Orquídeas silvestres. Diputación de Córdoba, Córdoba.

Sáez, L., Quijada, M.P., Alarcón M.L. \& Aldasoro, J.J. (2005). Serapias L. En S. Castroviejo, C. Aedo \& A. Herrero (Eds.), Flora Ibérica vol. XXI (pp. 164). Madrid: Real Jardín Botánico, CSIC

Silvestre, S. (1987) Serapias, Orchidaceae. En B. Valdés, S. Talavera \& E.F. Galiano (Eds.), Flora Vascular de Andalucía occidental vol III (pp. 511-512). Barcelona: Ketres. 